\title{
LOCALISATION AND COLOCALISATION OF TRIANGULATED CATEGORIES AT THICK SUBCATEGORIES
}

\author{
HVEDRI INASSARIDZE, TAMAZ KANDELAKI and RALF MEYER*
}

\begin{abstract}
Given a thick subcategory of a triangulated category, we define a colocalisation and a natural long exact sequence that involves the original category and its localisation and colocalisation at the subcategory. Similarly, we construct a natural long exact sequence containing the canonical map between a homological functor and its total derived functor with respect to a thick subcategory.
\end{abstract}

\section{Introduction}

Throughout this article, $\mathscr{T}$ is a triangulated category and $\mathscr{E}$ is a thick subcategory. The localisation of $\mathscr{T}$ at $\mathscr{E}$ is a triangulated category $\mathscr{T} / \mathscr{E}$ with a triangulated functor $L: \mathscr{T} \rightarrow \mathscr{T} / \mathscr{E}$ such that $\left.L\right|_{\mathscr{E}} \cong 0$, and such that any other triangulated functor with this property factors uniquely through $L$. This universal property determines the localisation uniquely up to isomorphism (if it exists).

The localisation is constructed in [8], disregarding set-theoretic problems (morphism sets may be classes, not sets). The same problems also appear in our context. We assume that the colimits that we need exist. This follows, for instance, if the triangulated categories in question are essentially small.

Given an Abelian category $\mathscr{C}$ and a homological functor $F: \mathscr{T} \rightarrow \mathscr{C}$, its right localisation at $\mathscr{E}$ (in the notation of [6]) is a homological functor $\mathrm{R} F: \mathscr{T} \rightarrow \mathscr{C}$ together with a natural transformation $F \Rightarrow \mathrm{R} F$, such that $\mathrm{R} F$ vanishes on $\mathscr{E}$ and such that any other natural transformation $F \Rightarrow G$ for a homological functor $G$ with $\left.G\right|_{\mathscr{E}}=0$ factors uniquely through $F \Rightarrow \mathrm{R} F$. Left localisations are defined similarly, but they will not be treated here.

If $F$ is a homological functor to the category $\mathfrak{A} \mathfrak{b}$ of Abelian groups, then

\footnotetext{
* This research was supported by the Volkswagen Foundation (Georgian-German Non-Commutative Partnership). The third author was supported by the German Research Foundation (Deutsche Forschungsgemeinschaft (DFG)) through the Institutional Strategy of the University of Göttingen.

Received 7 September 2010, in final form 1 May 2011.
} 
we construct a functor $\mathrm{R}^{\perp} F$ that fits into a long exact sequence

$$
\begin{aligned}
\cdots \rightarrow \mathrm{R}^{\perp} F_{1}(A) \rightarrow F_{1}(A) & \rightarrow \mathrm{R} F_{1}(A) \\
& \rightarrow \mathrm{R}^{\perp} F_{0}(A) \rightarrow F_{0}(A) \rightarrow \mathrm{R} F_{0}(A) \rightarrow \cdots
\end{aligned}
$$

which is functorial in $A$ and $F$; that is, a natural transformation of homological functors $F \Rightarrow F^{\prime}$ and an arrow $A \rightarrow A^{\prime}$ induce a chain map from (1) for $F_{*}(A)$ to (1) for $F_{*}^{\prime}\left(A^{\prime}\right)$ in a functorial manner.

We call a homological functor $F: \mathscr{T} \rightarrow \mathfrak{U b}$ local if the natural map $F \Rightarrow \mathrm{R} F$ is invertible, and colocal if the map $\mathrm{R}^{\perp} F \Rightarrow F$ is invertible. As we shall explain, local homological functors correspond to homological functors $\mathscr{T} / \mathscr{E} \rightarrow \mathfrak{A b}$, while colocal homological functors correspond to homological functors $\mathscr{E} \rightarrow \mathfrak{U} \mathfrak{b}$. Thus (1) decomposes homological functors on $\mathscr{T}$ into homological functors on $\mathscr{T} / \mathscr{E}$ and $\mathscr{E}$.

Dual statements, with left and right exchanged, apply to cohomological functors $\mathscr{T} \rightarrow \mathfrak{U} \mathfrak{b}$ if we view them as homological functors $\mathscr{T}^{\text {op }} \rightarrow \mathfrak{U} \mathfrak{b}$ on the opposite category $\mathscr{T}^{\text {op }}$.

Let us specialise (1) to the functor $\mathscr{T}(A, \sqcup)$ for an object $A$ of $\mathscr{T}$. Let $A[n]$ denote the $n$-fold suspension of $A$ and let $\mathscr{T}_{n}(A, B):=\mathscr{T}(A, B[n]) \cong$ $\mathscr{T}(A[-n], B)$, and similarly for $\mathscr{T} / \mathscr{E}$. Our construction embeds the localisation functor $L: \mathscr{T} \rightarrow \mathscr{T} / \mathscr{E}$ into a natural long exact sequence

$$
\begin{aligned}
\cdots \rightarrow & \mathscr{T} / \mathscr{E}_{1}^{\perp}(A, B) \rightarrow \mathscr{T}_{1}(A, B) \stackrel{L}{\longrightarrow} \mathscr{T} / \mathscr{E}_{1}(L A, L B) \\
& \rightarrow \mathscr{T} / \mathscr{E}_{0}^{\perp}(A, B) \rightarrow \mathscr{T}_{0}(A, B) \stackrel{L}{\longrightarrow} \mathscr{T} / \mathscr{E}_{0}(L A, L B) \rightarrow \cdots
\end{aligned}
$$

with $\mathscr{T} / \mathscr{E}_{n}^{\perp}(A, B):=\mathscr{T} / \mathscr{E}^{\perp}(A, B[n]) \cong \mathscr{T} / \mathscr{E}^{\perp}(A[-n], B)$. We call $\mathscr{T} / \mathscr{E}^{\perp}$ the colocalisation of $\mathscr{T}$ at $\mathscr{E}$. The naturality of (2) means that $\mathscr{T} / \mathscr{E} \perp(A, B)$ is a bifunctor on $\mathscr{T}$ contravariant in the first and covariant in the second variable, and that the maps in (2) are natural transformations. Using the natural transformation $\mathscr{T} / \mathscr{E} \perp \rightarrow \mathscr{T}$ in (2) and the $\mathscr{T}$-bimodule structure, $\mathscr{T} / \mathscr{E} \perp$ is equipped with an associative composition product. It is not a category because it lacks identity morphisms.

The exact sequences (1) and (2) are easy to construct in the special situation of a complementary pair of thick subcategories in the notation of [7]. (In [8], this is called a Bousfield localisation, and [2] speaks of admissible subcategories.) Recall that $(\mathscr{E} \perp, \mathscr{E})$ is a complementary pair of thick subcategories if $\mathscr{T}(\mathscr{E} \perp, \mathscr{E})=0$ and for any object $B$ of $\mathscr{T}$ there is an exact triangle

$$
L^{\perp} B \rightarrow B \rightarrow L B \rightarrow L^{\perp} B[1]
$$

with $L B \in \in \mathscr{E}^{\perp}, L^{\perp} B \in \in \mathscr{E}$. Here we write $\in \in$ for objects of categories, as opposed to $\in$ for morphisms of categories. The triangle (3) is unique up to 
canonical isomorphism and depends functorially on $B$. Since

$$
\mathscr{T} / \mathscr{E}(A, B) \cong \mathscr{T}(A, L B) \cong \mathscr{T}(L A, L B), \quad \mathrm{R} F(B) \cong F(L B),
$$

we may define

$$
\mathscr{T} / \mathscr{E}^{\perp}(A, B):=\mathscr{T}\left(A, L^{\perp} B\right), \quad \mathrm{R}^{\perp} F(B):=F\left(L^{\perp} B\right) .
$$

Then the exact sequences (1) and (2) follow by applying the homological functors $\mathscr{T}\left(A, \_\right)$and $F$ to the exact triangle (3).

Without $\mathscr{E} \perp$, we cannot single out a unique exact triangle as in (3). Instead, we construct the localisation and the colocalisation as colimits of $\mathscr{T}(A, D)$ and $\mathscr{T}(A, C)$, where $C$ and $D$ are indexed by the category of exact triangles $C \rightarrow B \rightarrow D \rightarrow C[1]$ with $C \in \in \mathscr{E}$. The main issue is to prove that this category of exact triangles is filtered, so that the colimit preserves exact sequences.

A typical example where the general construction of the colocalisation is useful is considered in [5]. There $\mathscr{T}(A, B)=\mathrm{KK}(A, B)$ is Kasparov's bivariant K-theory for $\mathrm{C}^{*}$-algebras $A$ and $B$, and $\mathscr{T} / \mathscr{E}(A, B)=\mathrm{KK}(A, B) \otimes \mathrm{Q}$. The colocalisation provides a torsion variant of KK-theory. It can be shown that $\mathscr{T} / \mathscr{E}$ is the localisation of $\mathrm{KK}$ at a suitable thick subcategory. It is unclear, however, whether this is part of a complementary pair of thick subcategories.

A previous instance of the localisation-colocalisation exact sequence appears in [3, Theorem 6.2.5(3)]. Implicitly, such an exact sequence is also used in the study of noncommutative projective schemes [1].

This article is organised as follows. Section 2 recalls the well-known construction of the localisation and its basic properties. Similar techniques are used in Section 3 to define and study colocalisations. Section 4 establishes the exact sequences (1) and (2). Each section uses a particular filtered category.

\section{Localisation}

We fix a triangulated category $\mathscr{T}$ and a thick subcategory $\mathscr{E}$ throughout. We assume that the colimits of sets in (4) below exist; this follows if $\mathscr{T}$ is essentially small.

The cone of a morphism $f: A \rightarrow B$ in $\mathscr{T}$ is the object $C$ in an exact triangle

$$
A \stackrel{f}{\longrightarrow} B \rightarrow C \rightarrow A[1] .
$$

A morphism $f$ in $\mathscr{T}$ is an $\mathscr{E}$-weak equivalence if its cone belongs to $\mathscr{E}$. Let we $\mathscr{E}(A, B)$ be the set of $\mathscr{E}$-weak equivalences from $A$ to $B$.

Recall that an object of $\mathscr{T}$ becomes zero in $\mathscr{T} / \mathscr{E}$ if and only if it belongs to $\mathscr{E}$, and an arrow in $\mathscr{T}$ becomes invertible in $\mathscr{T} / \mathscr{E}$ if and only if it is an $\mathscr{E}$-weak 
equivalence (see [8]). Thus we $\mathrm{E}_{\mathscr{E}}$ is closed under composition and contains all isomorphisms in $\mathscr{T}$. Even more, we $\mathscr{E}_{\mathscr{E}}$ has the two-out-of-three and even the two-out-of-six property:

Lemma 2.1. Let $f, g$ and $h$ be composable morphisms in $\mathscr{T}$. If $f g$ and $g h$ are $\mathscr{E}$-weak equivalences, so are $f, g, h$, and $f g h$.

If two of $f, g$, and $f g$ are $\mathscr{E}$-weak equivalences, so is the third.

Proof. Let $L: \mathscr{T} \rightarrow \mathscr{T} / \mathscr{E}$ be the canonical functor to the localisation. The assumption means that $L(f g)$ and $L(g h)$ are invertible in $\mathscr{T} / \mathscr{E}$. This implies that $L(g)$ has both a left and a right inverse, so that $L(g)$ is invertible. Then $L(f)$ and $L(h)$ must also be invertible, and finally $L(f g h)$ is invertible. The second statement follows from the first one by considering the special cases where one of $f, g$ or $h$ is an identity (see also [8, Lemma 1.5.6] for a more direct proof of the two-out-of-three property).

The construction of the localisation $\mathscr{T} / \mathscr{E}$ in [8] shows that its underlying category is isomorphic to the localisation of $\mathscr{T}$ at we $\mathscr{E}$ in the category theoretic sense of [4]. To prepare for this, it is shown that we $\mathscr{E}$ allows a calculus of left fractions in the sense of $[4, \S 2.2]$, that is, it satisfies the following conditions (LF1)-(LF3):

LEMMA 2.2. Let $\mathscr{E}$ be a thick subcategory of a triangulated category $\mathscr{T}$.

(LF1) Identities belong to $\mathrm{we}_{\mathscr{E}}$, and $\mathrm{we}_{\mathscr{E}}$ is closed under composition.

(LF2) For each pair of morphisms $B \stackrel{s}{\longleftarrow} A \stackrel{f}{\longrightarrow} C$ with $s \in$ we $_{\mathscr{E}}$ there are $g \in \mathscr{T}$ and $t \in \mathrm{we}_{\mathscr{E}}$ with $g s=t f$, that is, there is a commuting square

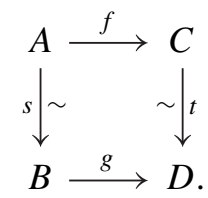

(LF3) If $f, g: A \Rightarrow B$ are parallel morphisms in $\mathscr{T}$ and $s: A^{\prime} \rightarrow A$ is in we and satisfies $f s=g s$, then there is $t: B \rightarrow B^{\prime}$ in weש̋ with $t f=t g$.

Proof. (LF1) is obvious, and (LF2) follows from [8, Lemma 1.5.8] and the Octahedral Axiom. We only have to prove (LF3). We construct the following commuting diagram:

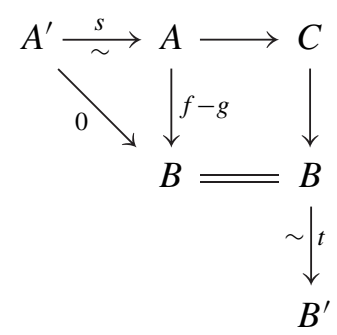


First we embed $s: A^{\prime} \rightarrow A$ in an exact triangle $A^{\prime} \stackrel{s}{\longrightarrow} A \rightarrow C \rightarrow A^{\prime}[1]$. Then $C \in \in \mathscr{E}$ because $s \in$ we $\mathscr{E}$. Since $(f-g) \circ s=0$ and $\mathscr{T}(\lrcorner, B)$ is cohomological, we may factor $f-g$ through the map $A \rightarrow C$. We embed the resulting map $C \rightarrow B$ into an exact triangle $C \rightarrow B \stackrel{t}{\rightarrow} B^{\prime} \rightarrow C$ [1]. Since $C \in \in \mathscr{E}, t \in$ we $_{\mathscr{E}}$. By construction $t \circ(f-g)=0$.

Dually, there is a calculus of right fractions for the same reasons.

It is useful for us to rewrite $\mathscr{T} / \mathscr{E}(A, B)$ as a filtered colimit. For a fixed object $B \in \in \mathscr{T}$, we consider the category $B \downarrow$ we $\mathscr{E}$ whose objects are arrows $B \rightarrow C$ in $\mathrm{we}_{\mathscr{E}}$ and whose arrows are commuting triangles:

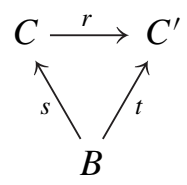

where $s$ and $t$ are morphisms in we $\mathscr{E}$ and $r$ is a morphism in $\mathscr{T}$. By the twoout-of-three property, we also get $r \in$ we $\mathscr{E}$.

Recall that a category is called filtered if it satisfies the following conditions:

(F1) for two objects $E$ and $D$ there are an object $H$ and morphisms $f: E \rightarrow H$ and $g: D \rightarrow H$;

(F2) for a pair of parallel morphisms $h, j: A \Rightarrow B$ there is a morphism $l: B \rightarrow$ $F$ with $l h=l j$.

Since we w $_{\mathscr{E}}$ allows a calculus of left fractions by Lemma 2.2, the category $B \downarrow$ we $\mathscr{E}$ is filtered for each $B$. (The proof that (LF1)-(LF3) imply (F1) and (F2) is an easy exercise.)

The map $s \mapsto \mathscr{T}(A, C)$ for $(s: B \rightarrow C) \in \in B \downarrow$ we w $_{\mathscr{E}}$ defines a covariant functor from $B \downarrow$ we $_{\mathscr{E}}$ to the category of sets. The colimit of this diagram yields the space of arrows $\mathscr{T} / \mathscr{E}(A, B)$ in the localisation of $\mathscr{T}$ at we $\mathscr{E}$ :

$$
\mathscr{T} \mid \mathscr{E}(A, B):=\lim _{(s: B \rightarrow C) \in \in\left(B \downarrow \mathrm{we}_{\mathscr{E}}\right)} \mathscr{T}(A, C) .
$$

The existence of these colimits for all $A$ and $B$ ensures that the localisation exists. Recall that any small diagram of Abelian groups has a colimit. If $\mathscr{T}$ is (essentially) small, then so is $B \downarrow$ we $\mathscr{E}$, and hence the colimit in (4) exists. There are many examples where $\mathscr{T}$ is large but the colimits in (4) exist for different reasons.

For instance, if $\mathscr{E}$ is part of a complementary pair of thick subcategories $(\mathscr{E} \perp, \mathscr{E})$ in the notation of [7], then the arrow $B \rightarrow L B$ in (3) is a final object of $B \downarrow$ we $\mathscr{E}$. Thus the colimit above exists and reduces to $\mathscr{T} / \mathscr{E}(A, B) \cong$ 
$\mathscr{T}(A, L B)$. Conversely, if $B \downarrow$ we $\mathscr{E}$ has a final object for each $B$, then $(\mathscr{E} \perp, \mathscr{E})$ is a complementary pair of thick subcategories.

Next we want to prove that the inductive system $(C)_{s: B \rightarrow C \in \in B \downarrow w_{\mathscr{E}}}$ depends functorially on $B$. For this, we recall the standard notion of a morphism between two inductive systems in the same category. Let $\mathscr{D}_{1}$ and $\mathscr{D}_{2}$ be filtered categories and let $F_{1}: \mathscr{D}_{1} \rightarrow \mathscr{C}$ and $F_{2}: \mathscr{D}_{2} \rightarrow \mathscr{C}$ be functors. Then

$$
\operatorname{Hom}\left(F_{1}, F_{2}\right):=\lim _{x_{1} \in \in \mathscr{D}_{1}} \underset{x_{2} \in \in \mathscr{D}_{2}}{\lim _{\overrightarrow{1}}} \mathscr{C}\left(F_{1}\left(x_{1}\right), F_{2}\left(x_{2}\right)\right)
$$

Thus the data of a morphism of inductive systems from $F_{1}$ to $F_{2}$ is the following: for each $x_{1} \in \in \mathscr{D}_{1}$, we have $x_{2} \in \in \mathscr{D}_{2}$ and an arrow $F_{1}\left(x_{1}\right) \rightarrow F_{2}\left(x_{2}\right)$. These satisfy suitable compatibility conditions for varying $x_{1}$. More conceptually, morphisms are natural transformations between the associated functors,

$$
\left.\left.\lim _{x_{1} \in \in \mathscr{D}_{1}} \mathscr{C}(\lrcorner, F_{1}\left(x_{1}\right)\right) \rightarrow \underset{x_{2} \in \in \mathscr{D}_{2}}{\lim _{\overrightarrow{1}}} \mathscr{C}(\lrcorner, F_{2}\left(x_{2}\right)\right)
$$

LEMMA 2.3. The inductive system $(C)_{s: B \rightarrow C \in \in B \downarrow \text { we }_{\mathscr{E}}}$ depends functorially on $B$.

Proof. Let $f: B \rightarrow B^{\prime}$ be a morphism in $\mathscr{T}$. For an object $s: B \rightarrow C$ of $B \downarrow$ we $_{\mathscr{E}}$, (LF2) allows us to choose an object $t: B^{\prime} \rightarrow C^{\prime}$ of $B^{\prime} \downarrow$ we $_{\mathscr{E}}$ and a map $g: C \rightarrow C^{\prime}$ with $g s=t f$. When we view $g$ as an element of

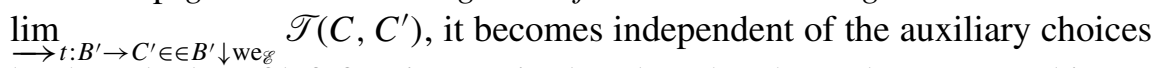
by the calculus of left fractions. It is also clear that these elements combine to one of

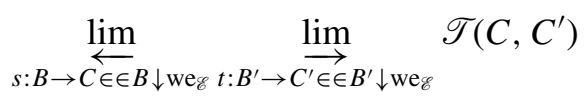

when we let $s$ vary. Hence they combine to a morphism of inductive systems.

Alternatively, morphisms of inductive systems $\left(C_{s}\right) \rightarrow\left(C_{s^{\prime}}^{\prime}\right)$ correspond bijectively to natural transformations $\mathscr{T}\left(X,\left(C_{s}\right)\right) \rightarrow \mathscr{T}\left(X,\left(C_{s^{\prime}}^{\prime}\right)\right)$. Since $\mathscr{T}\left(X,\left(C_{s}\right)\right)=\mathscr{T} / \mathscr{E}(X, B)$, our statement is equivalent to the existence of a natural composition product

$$
\mathscr{T} / \mathscr{E}(X, B) \times \mathscr{T}\left(B, B^{\prime}\right) \rightarrow \mathscr{T} / \mathscr{E}\left(X, B^{\prime}\right)
$$

This exists because $\mathscr{T} / \mathscr{E}$ is a category and the maps $\mathscr{T} \rightarrow \mathscr{T} / \mathscr{E}$ are a functor.

Definition 2.4. Let $F: \mathscr{T} \rightarrow \mathfrak{U} \mathfrak{b}$ be a homological functor to the category of Abelian groups. Its right derived functor or right localisation $\mathrm{R} F: \mathscr{T} \rightarrow \mathfrak{U b}$ is defined by

$$
\mathrm{R} F(B):=\lim _{(s: B \rightarrow C) \in \in B \downarrow \text { we } \mathscr{E}} F(C) .
$$


This is a functor by Lemma 2.3. More explicitly, represent an element of $\mathrm{R} F(B)$ by an $\mathscr{E}$-weak equivalence $s: B \rightarrow C$ and $x \in F(C)$; let $f: B \rightarrow B^{\prime}$ be a morphism. The calculus of fractions provides a commuting diagram

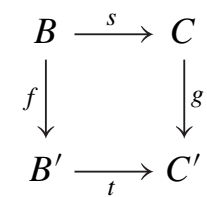

with an $\mathscr{E}$-weak equivalence $t$; then $\left[t, g_{*}(x)\right]$ represents $f_{*}[s, x]$.

The maps $F(B) \rightarrow \mathrm{R} F(B), x \mapsto\left[\operatorname{id}_{B}, x\right]$, form a natural transformation $F \Rightarrow \mathrm{R} F$.

As is well-known, the definition above does not use the triangulated category structure, it produces the localisation in the usual sense of category theory (see also [6]).

THEOREM 2.5. Let $\mathscr{T}$ be a triangulated category, $\mathscr{E}$ a thick subcategory, and $F: \mathscr{T} \rightarrow \mathfrak{U} \mathfrak{b}$ a homological functor. Then the right localisation $\mathrm{R} F: \mathscr{T} \rightarrow \mathfrak{T H}$ is homological.

Proof. Let $B^{\prime} \stackrel{f}{\longrightarrow} B \stackrel{g}{\longrightarrow} B^{\prime \prime} \rightarrow B^{\prime}[1]$ be an exact triangle. We must show that

$$
\mathrm{R} F\left(B^{\prime}\right) \stackrel{\mathrm{R} F(f)}{\longrightarrow} \mathrm{R} F(B) \stackrel{\mathrm{R} F(g)}{\longrightarrow} \mathrm{R} F\left(B^{\prime \prime}\right)
$$

is an exact sequence of groups. Functoriality of $\mathrm{R} F$ already implies $\operatorname{im} \mathrm{R} F(f) \subseteq \operatorname{ker} \mathrm{R} F(g)$. It remains to prove $\operatorname{im} \mathrm{R} F(f) \supseteq \operatorname{ker} \mathrm{R} F(g)$.

Let $s \in$ we $_{\mathscr{E}}(B, C)$ and $x \in F(C)$ represent an element in $\operatorname{ker} \mathrm{R} F(g)$. By the definition of the functoriality of $\mathrm{R} F$, this means that there is a commuting diagram

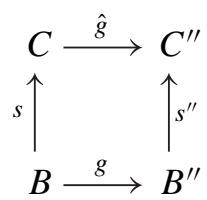

with $s^{\prime \prime} \in$ we $_{\mathscr{E}}$ and $F(\hat{g})(x)=0$. By the properties of triangulated categories, we may complete this to a morphism of exact triangles

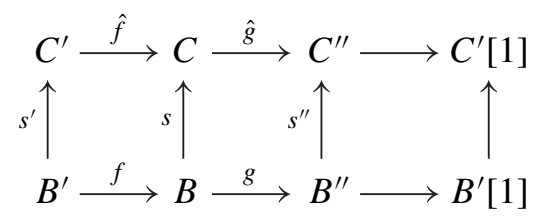


Since $s, s^{\prime \prime} \in$ we $_{\mathscr{E}}$, we also get $s^{\prime} \in$ we $_{\mathscr{E}}$ from the Octahedral Axiom. Since $F$ is homological, there is an element $x^{\prime} \in F\left(C^{\prime}\right)$ with $F(\hat{f})\left(x^{\prime}\right)=x$. Therefore, the class of the pair $\left(s^{\prime}, x^{\prime}\right)$ in $\mathrm{R} F\left(B^{\prime}\right)$ maps to the class of $(s, x)$.

Recall that the category $\mathscr{T}^{\mathrm{op}}$ is again triangulated, with new suspension automorphism $A \mapsto A[-1]$ and the same exact triangles: $A[1] \leftarrow C \leftarrow$ $B \leftarrow A$ is an exact triangle in $\mathscr{T}^{\text {op }}$ if $A \rightarrow B \rightarrow C \rightarrow A[1]$ is an exact triangle in $\mathscr{T}$. Thus we may view a cohomological functor $\mathscr{T} \rightarrow \mathfrak{U} \mathfrak{b}$ as a homological functor $\mathscr{T}^{\text {op }} \rightarrow \mathfrak{A} \mathfrak{h}$. Definition 2.4 applied to a homological functor $\mathscr{T}^{\mathrm{op}} \rightarrow \mathfrak{U} \mathfrak{W}$ yields a right localisation for the original cohomological functor $\mathscr{T} \rightarrow \mathfrak{H} \mathfrak{b}$.

Theorem 2.5 may be extended to the case where $F$ is a homological functor with values in an Abelian category $\mathscr{C}$ with exact filtered colimits. However, some assumption on $\mathscr{C}$ seems necessary. In particular, it is unclear how to treat homological functors $\mathscr{T} \rightarrow \mathfrak{A} \mathfrak{b}^{\text {op }}$ because filtered colimits in $\mathfrak{A} \mathfrak{b}^{\mathrm{op}}$ are filtered limits in $\mathfrak{U} \mathfrak{b}$, and these need not be exact.

By definition, the functor $B \mapsto \mathscr{T} / \mathscr{E}(A, B)$ is the right localisation of $B \mapsto \mathscr{T}(A, B)$. Therefore, this functor is homological by Theorem 2.5. Since filtered colimits are exact, the functor $A \mapsto \mathscr{T} / \mathscr{E}(A, B)$ is cohomological by (4). Of course, this also follows from the stronger statement that $\mathscr{T} / \mathscr{E}$ is a triangulated category.

We may also apply the construction above to get the localisation of $\mathscr{T}^{\mathrm{op}}$ at $\mathscr{E} O$ p. The opposite category of this localisation provides another model for $\mathscr{T} / \mathscr{E}$ that is based on a calculus of right fractions instead of left fractions. Both constructions agree because both localisations share the same universal property.

Proposition 2.6. Let F: $\mathscr{T} \rightarrow \mathfrak{d} \mathfrak{b}$ be a homological functor. The following assertions are equivalent:

(a) the natural transformation $F \Rightarrow \mathrm{R} F$ is invertible;

(b) $F(E) \cong 0$ for all $E \in \in \mathscr{E}$;

(c) $F(s)$ is invertible for all $s \in$ we $_{\mathscr{E}}$;

(d) F factors through a homological functor $\mathscr{T} / \mathscr{E} \rightarrow \mathfrak{U}$ b.

Furthermore, $\mathrm{R} F$ always satisfies these equivalent conditions.

Proof. If $E \in \in \mathscr{E}$, then the zero map $E \rightarrow 0$ is an $\mathscr{E}$-weak equivalence. It is a final object in $E \downarrow$ we $_{\mathscr{E}}$, so that $\mathrm{R} F(E)=F(0)=0$. Thus $\mathrm{R} F(E)=0$ always satisfies (b), and hence (a) implies (b). If $s \in$ we $_{\mathscr{E}}$, then $s$ is part of an exact triangle $A \stackrel{s}{\longrightarrow} B \rightarrow E \rightarrow A[1]$ with $E \in \in \mathscr{E}$. The long exact sequence for $F$ applied to this exact triangle shows that $F_{*}(s)$ is invertible if and only if $F_{*}(E) \cong 0$. Since we $\mathscr{E}$ and $\mathscr{E}$ are closed under suspensions, this 
yields (b) $\Leftrightarrow($ c). Since $\mathscr{T} / \mathscr{E}$ is the localisation of $\mathscr{T}$ at we $\mathscr{E}$ in the sense of category theory, a functor on $\mathscr{T}$ factors through $\mathscr{T} / \mathscr{E}$ if and only if it maps all arrows in we $\mathscr{E}$ to invertible arrows. Furthermore, the functor on $\mathscr{T} / \mathscr{E}$ induced in this way is again homological if $F$ was (see [8]). Thus (c) $\Leftrightarrow$ (d). Finally, (c) implies that the maps $F(B) \rightarrow F(C)$ are invertible for all $s: B \rightarrow C$ in $B \downarrow$ we $\mathscr{E}$. Thus (c) implies (a).

There is an analogous result for cohomological functors.

A (co)homological functor with the equivalent properties in Proposition 2.6 is called local. Condition (d) means that local (co)homological functors $\mathscr{T} \rightarrow$ $\mathfrak{U} \mathfrak{b}$ are equivalent to local (co)homological functors $\mathscr{T} / \mathscr{E} \rightarrow \mathfrak{U} \mathfrak{b}$.

Proposition 2.7. The localisation $\mathrm{R} F$ is the universal local homological functor on $\mathscr{T}$ equipped with a natural transformation $F \Rightarrow \mathrm{R} F$ : if $G$ is any local homological functor on $\mathscr{T}$, then there is a natural bijection between natural transformations $F \Rightarrow G$ and natural transformations $\mathrm{R} F \Rightarrow G$.

This universal property characterises $\mathrm{R} F$ uniquely up to natural isomorphism.

Proof. If $G$ is local, then a natural transformation $\Phi: F \Rightarrow G$ induces a natural transformation $\Phi^{\prime}: \mathrm{R} F \Rightarrow \mathrm{R} G \cong G$. The composition of $\Phi^{\prime}$ with the natural transformation $\Psi: F \Rightarrow \mathrm{R} F$ is again $\Phi$, and $\Phi^{\prime}$ is the only natural transformation $\mathrm{R} F \Rightarrow G$ with $\Phi^{\prime} \circ \Psi=\Phi$.

\section{Colocalisation}

Let $\mathscr{E} \downarrow B$ be the category, whose objects are arrows $f: E \rightarrow B$ with $E \in \in \mathscr{E}$ and whose arrows are commuting triangles

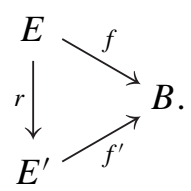

Lemma 3.1. The category $\mathscr{E} \downarrow B$ is filtered for all $B \in \in \mathscr{T}$.

Proof. The first axiom (F1) of a filtered category follows easily because $\mathscr{E}$ is additive: any pair of maps $E_{1} \rightarrow B, E_{2} \rightarrow B$ is dominated by $E_{1} \oplus E_{2} \rightarrow B$. To verify (F2), we must equalise a diagram of the form

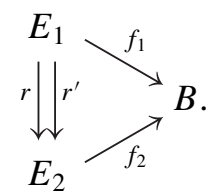


Embed $r-r^{\prime}$ in an exact triangle $E_{1} \stackrel{r-r^{\prime}}{\longrightarrow} E_{2} \rightarrow E_{3} \rightarrow E_{1}[1]$. Since $f_{2} \circ$ $\left(r-r^{\prime}\right)=0$ and $\mathscr{T}(-, B)$ is cohomological, we may factor $f_{2}$ through the map $E_{2} \rightarrow E_{3}$. This yields a commuting diagram

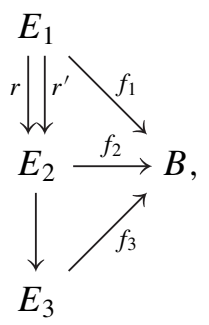

which equalises the diagram (5) in $\mathscr{E} \downarrow B$.

Definition 3.2. The colocalisation of $\mathscr{T}$ at $\mathscr{E}$ is defined by

$$
\mathscr{T} / \mathscr{E}^{\perp}(A, B):=\lim _{(s: C \rightarrow B) \in \in \mathscr{E} \downarrow B} \mathscr{T}(A, C) .
$$

This is a bifunctor in the obvious way: a map $f: A \rightarrow A^{\prime}$ acts by composition $\mathscr{T}\left(A^{\prime}, C\right) \rightarrow \mathscr{T}(A, C)$ for all $C$; a map $g: B \rightarrow B^{\prime}$ maps a pair $(h, s)$ with $s: C \rightarrow B$ in $\mathscr{E} \downarrow B$ and $h \in \mathscr{T}(A, C)$ to $(h, g \circ s)$.

As in the discussion after (4), this colimit exists if $\mathscr{T}$ is small or if $\mathscr{E} \downarrow B$ has a final element. The latter happens for all $B$ if and only if $\mathscr{E}$ is part of a complementary pair of thick subcategories $(\mathscr{E} \perp, \mathscr{E})$. In this case, the map $L^{\perp} B \rightarrow B$ in (3) is a final object in $\mathscr{E} \downarrow B$, so that $\mathscr{T} / \mathscr{E} \perp(A, B) \cong \mathscr{T}\left(A, L^{\perp} B\right)$.

We will see below that Definition 3.2 leads to an exact sequence as in (2).

The naturality of the inductive system $(C)_{s \in \in \mathscr{E} \downarrow}$ is trivial: an arrow $f: B \rightarrow$ $B^{\prime}$ in $\mathscr{T}$ induces a morphism of inductive systems

$$
(C)_{(s: C \rightarrow B) \in \in \mathscr{E} \downarrow B} \rightarrow\left(C^{\prime}\right)_{\left(s^{\prime}: C^{\prime} \rightarrow B^{\prime}\right) \in \in \mathscr{E} \downarrow B^{\prime}},
$$

which maps $s: C \rightarrow B$ to $f \circ s: C \rightarrow B^{\prime}$ and acts identically on $C$. As a result, $B \mapsto \mathscr{T} / \mathscr{E} \perp(A, B)$ is a bifunctor that is contravariant in $A$ and covariant in $B$.

The maps $s: C \rightarrow B$ provide a natural natural transformation

$$
\mathscr{T} / \mathscr{E}^{\perp}(A, B) \rightarrow \mathscr{T}(A, B) .
$$

We may describe elements of $\mathscr{T} / \mathscr{E} \perp(A, B)$ as diagrams $A \stackrel{f}{\longrightarrow} \tilde{B} \stackrel{s}{\longrightarrow} B$ with $\tilde{B} \in \in \mathscr{E}$. The natural map to $\mathscr{T}(A, B)$ maps this diagram to $s f: A \rightarrow B$.

The naturality $\mathscr{T}(B, C) \times \mathscr{T} / \mathscr{E} \perp(A, B) \rightarrow \mathscr{T} / \mathscr{E} \perp(A, C)$ and the natural map $\mathscr{T} / \mathscr{E} \perp(B, C) \rightarrow \mathscr{T}(B, C)$ provide a multiplication

$$
\mathscr{T} / \mathscr{E}^{\perp}(B, C) \times \mathscr{T} / \mathscr{E} \perp(A, B) \rightarrow \mathscr{T} / \mathscr{E}^{\perp}(A, C) .
$$


The product of $B \stackrel{f_{1}}{\longrightarrow} \tilde{C} \stackrel{s_{1}}{\longrightarrow} C$ and $A \stackrel{f_{2}}{\longrightarrow} \tilde{B} \stackrel{s_{2}}{\longrightarrow} B$ is

$$
A \stackrel{f_{1} s_{2} f_{2}}{\longrightarrow} \tilde{C} \stackrel{s_{1}}{\longrightarrow} C \sim A \stackrel{f_{2}}{\longrightarrow} \tilde{B} \stackrel{s_{1} f_{1} s_{2}}{\longrightarrow} C,
$$

where $\sim$ denotes equivalence in the inductive limit $\mathscr{T} / \mathscr{E} \perp(A, C)$. That is, we get the same multiplication on $\mathscr{T} / \mathscr{E}{ }^{\perp}$ if we use the right multiplication map

$$
\mathscr{T} / \mathscr{E}^{\perp}(B, C) \times \mathscr{T}(A, B) \rightarrow \mathscr{T} / \mathscr{E}^{\perp}(A, C)
$$

and the natural map $\mathscr{T} / \mathscr{E} \perp(A, B) \rightarrow \mathscr{T}(A, B)$. It is also straightforward to see that the multiplication on $\mathscr{T} / \mathscr{E} \perp$ is associative. However, $\mathscr{T} / \mathscr{E} \perp$ has no identity maps, so that it is not a category.

Definition 3.3. Given a homological functor $F: \mathscr{T} \rightarrow \mathfrak{U} \mathfrak{b}$ to the category of Abelian groups, we define its right colocalisation at $\mathscr{E}$ by

$$
\mathrm{R}^{\perp} F(B):=\lim _{(s: C \rightarrow B) \in \in \mathscr{E} \downarrow B} F(C) .
$$

This is a functor by composition: the map induced by $f: B \rightarrow B^{\prime}$ maps $(h, s)$ with $h \in F(C), s \in \in \mathscr{E} \downarrow B$ to $(h, f \circ s)$.

The naturality of the inductive system $(C)_{s \in \in \mathscr{E} \downarrow B}$ implies that $\mathrm{R}^{\perp} F$ is a functor on $\mathscr{T}$. The maps $s: C \rightarrow B$ induce a natural map $\mathrm{R}^{\perp} F(B) \rightarrow F(B)$. A natural transformation $F \Rightarrow F^{\prime}$ clearly induces a natural transformation $\mathrm{R}^{\perp} F \Rightarrow \mathrm{R}^{\perp} F^{\prime}$.

THEOREM 3.4. If $F$ is a homological functor on $\mathscr{T}$, then so is $\mathrm{R}^{\perp} F$.

Proof. Let $B^{\prime} \stackrel{f}{\longrightarrow} B \stackrel{g}{\longrightarrow} B^{\prime \prime} \rightarrow B^{\prime}[1]$ be an exact triangle. We must show that

$$
\mathrm{R}^{\perp} F\left(B^{\prime}\right) \stackrel{\mathrm{R}^{\perp} F(f)}{\longrightarrow} \mathrm{R}^{\perp} F(B) \stackrel{\mathrm{R}^{\perp} F(g)}{\longrightarrow} \mathrm{R}^{\perp} F\left(B^{\prime \prime}\right)
$$

is an exact sequence. Functoriality already implies $\operatorname{ker} \mathrm{R}^{\perp} F(g) \supseteq \operatorname{im} \mathrm{R}^{\perp} F(f)$. It remains to show ker $\mathrm{R}^{\perp} F(g) \subseteq \operatorname{im} \mathrm{R}^{\perp} F(f)$.

Represent an element of $\mathrm{R}^{\perp} F(B)$ by a pair $(s, x)$ with $E \in \in \mathscr{E}, s: E \rightarrow B$ and $x \in F(E)$. If $\mathrm{R}^{\perp} F(g)(s, x)=0$, then there is a commuting diagram

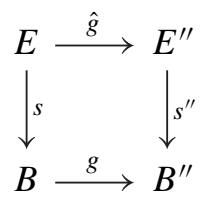


with $E^{\prime \prime} \in \in \mathscr{E}$ such that $F(\hat{g})(x)=0$. We may embed the above commuting square into a morphism of exact triangles

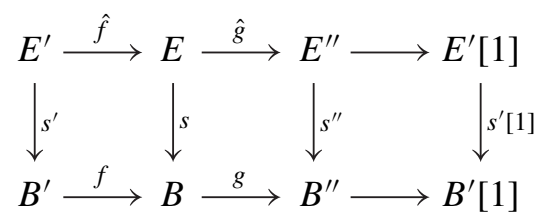

Since $F$ is homological and $F(\hat{g})(x)=0$, there is $x^{\prime} \in F\left(E^{\prime}\right)$ with $x=$ $F(\hat{f})\left(x^{\prime}\right)$. Thus the class of $\left(s^{\prime}, x^{\prime}\right)$ in $\mathrm{R}^{\perp} F\left(B^{\prime}\right)$ is a pre-image for the class of $(s, x)$.

THEOREM 3.5. The map $(A, B) \mapsto \mathscr{T} / \mathscr{E} \perp(A, B)$ defines a bifunctor that is cohomological in the first and homological in the second variable.

Proof. Since $\mathscr{T} / \mathscr{E} \perp(A\lrcorner$,$) is the right colocalisation of \mathscr{T}\left(A,{ }_{\lrcorner}\right)$, Theorem 3.4 shows that $\mathscr{T} / \mathscr{E} \perp(A, B)$ is homological in the second variable. It is cohomological in the first variable because filtered colimits are exact.

Definition 3.6. We call a (co)homological functor $F: \mathscr{T} \rightarrow \mathfrak{U} \mathfrak{b}$ colocal if the natural transformation $\mathrm{R}^{\perp} F \rightarrow F$ is invertible.

Proposition 3.7. Let $F: \mathscr{E} \rightarrow \mathfrak{U} \mathfrak{b}$ be a homological functor. Then there is a unique colocal homological functor $\bar{F}: \mathscr{T} \rightarrow$ Utb that extends $F$. Thus colocal homological functors $\mathscr{T} \rightarrow \mathfrak{U} \mathfrak{b}$ are essentially equivalent to homological functors $\mathscr{E} \rightarrow \mathfrak{U} \mathfrak{b}$.

Furthermore, $\mathrm{R}^{\perp} G$ is colocal for any homological functor $G: \mathscr{T} \rightarrow \mathfrak{U} \mathfrak{b}$. The natural transformation $\mathrm{R}^{\perp} G \Rightarrow G$ is universal among natural transformations from colocal functors to $G$.

Proof. We extend $F: \mathscr{E} \rightarrow \mathfrak{A} \mathfrak{b}$ by

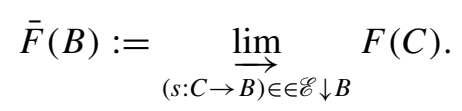

The proof of Theorem 3.4 only needs that $\left.F\right|_{\mathscr{E}}$ is a homological functor. Hence it shows that $\bar{F}$ is a homological functor.

Let $G: \mathscr{T} \rightarrow \mathfrak{U} \mathfrak{b}$ be any homological functor. If $B \in \in \mathscr{E}$, then $\operatorname{id}_{B}: B \rightarrow B$ is a final object in $\mathscr{E} \downarrow B$, so that we get $\mathrm{R}^{\perp} G(B) \cong G(B)$. In particular, this shows that $\left.\bar{F}\right|_{\mathscr{E}}=F$. Hence $\mathrm{R}^{\perp}\left(\mathrm{R}^{\perp} G\right) \cong \mathrm{R}^{\perp} G$ and $\mathrm{R}^{\perp} G$ is colocal. The definition of the colocalisation $\mathrm{R}^{\perp} G$ shows that two colocal functors that agree on $\mathscr{E}$ already agree on all of $\mathscr{T}$.

If $H$ is any colocal homological functor with a natural transformation $H \Rightarrow G$, then we get an induced natural transformation $H \cong \mathrm{R}^{\perp} H \Rightarrow \mathrm{R}^{\perp} G$. 
It is straightforward to see that this provides a bijection between natural transformations $H \Rightarrow G$ and $H \Rightarrow \mathrm{R}^{\perp} G$.

Remark 3.8. Furthermore, it can be shown that the colocalisation of $F$ at $\mathscr{E}$ is the left Kan extension of the restriction of $F$ to $\mathscr{E}$ along the inclusion $\mathscr{E} \rightarrow \mathscr{T}$.

\section{The localisation-colocalisation exact sequence}

To relate the localisation and colocalisation of $\mathscr{T}$ at $\mathscr{E}$, we introduce a third filtered category $\triangle_{\mathscr{E}} B$ that combines $B \downarrow$ we $\mathscr{E}$ and $\mathscr{E} \downarrow B$ for an object $B$ of $\mathscr{T}$. Objects of $\triangle_{\mathscr{C}} B$ are exact triangles of the form

$$
E \rightarrow B \stackrel{s}{\longrightarrow} C \rightarrow E[1]
$$

with $E \in \in \mathscr{E}$ or, equivalently, $s \in$ we $\mathscr{E}$; arrows in $\triangle_{\mathscr{E}} B$ are morphisms of triangles of the form

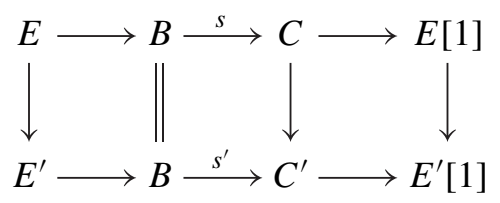

There are obvious forgetful functors from $\triangle_{\mathscr{E}} B$ to $B \downarrow$ we $\mathscr{\mathscr { E }}$ and $\mathscr{E} \downarrow B$ that extract the map $B \rightarrow C$ or the map $E \rightarrow B$, respectively. Since $s \in$ we $_{\mathscr{E}}$ if and only if $E \in \in \mathscr{E}$, any object of $B \downarrow$ we $\mathscr{E}$ or $\mathscr{E} \downarrow B$ is in the range of this forgetful functor. The axiom (TR3) for triangulated categories implies that these two forgetful functors are surjective on arrows as well.

Proposition 4.1. The category $\triangle_{\mathscr{E}} B$ is filtered.

Proof. First we check (F1). Consider two exact triangles

$$
\Delta:=(E \rightarrow B \stackrel{s}{\longrightarrow} C \rightarrow E[1]), \quad \Delta^{\prime}:=\left(E^{\prime} \rightarrow B \stackrel{s^{\prime}}{\longrightarrow} C^{\prime} \rightarrow E^{\prime}[1]\right)
$$

with $E, E^{\prime} \in \in \mathscr{E}$ and $s, s^{\prime} \in$ we $\mathscr{\mathscr { E }}$. (LF2) yields a commuting diagram

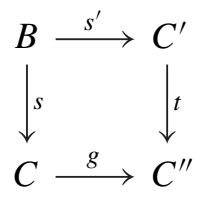

with $t \in$ we $_{\mathscr{E}}$. Then $t s^{\prime} \in$ we $_{\mathscr{E}}$ as well. We embed $t s^{\prime}$ in an exact triangle

$$
\Delta^{\prime \prime}:=\left(E^{\prime \prime} \rightarrow B \stackrel{t s^{\prime}}{\longrightarrow} C^{\prime \prime} \rightarrow E^{\prime \prime}[1]\right)
$$


this yields an object of $\triangle_{\mathscr{E}} B$. The axioms of a triangulated category yield morphisms

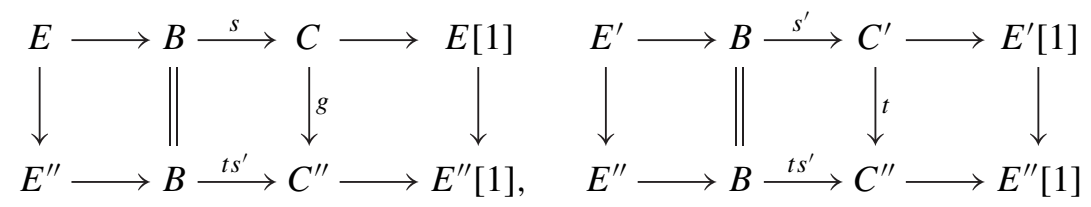

of triangles. Thus $\Delta^{\prime \prime}$ dominates both $\Delta$ and $\Delta^{\prime}$ in $\Delta_{\mathscr{E}} B$, verifying (F1).

Next we construct equalisers for parallel arrows in $\triangle_{\mathscr{E}} B$ :

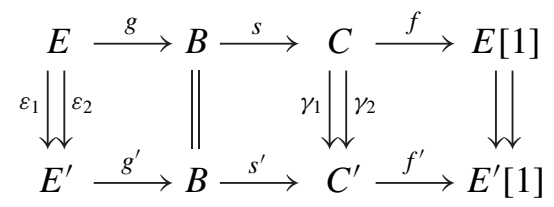

Since the category $B \downarrow$ we $\mathscr{E}$ is filtered, we may equalise $\gamma_{1}$ and $\gamma_{2}$ by a morphism $\gamma_{3}$ in $B \downarrow$ we e $_{\mathscr{E}}$. This lifts to a morphism $\left(\varepsilon_{3}, \gamma_{3}\right)$ in $\triangle_{\mathscr{E}} B$ that equalises $\gamma_{1}$ and $\gamma_{2}$. Since the category $\mathscr{E} \downarrow B$ is filtered as well by Proposition 3.1, we may equalise $\varepsilon_{3} \circ \varepsilon_{1}$ and $\varepsilon_{3} \circ \varepsilon_{2}$ by a morphism $\varepsilon_{4}$ in $\mathscr{E} \downarrow B$, which once again lifts to a morphism $\left(\varepsilon_{4}, \gamma_{4}\right)$ in $\triangle_{\mathscr{E}} B$. Then $\left(\varepsilon_{4} \circ \varepsilon_{3}, \gamma_{4} \circ \gamma_{3}\right)$ is a morphism in $\triangle_{\mathscr{E}} B$ that equalises $\left(\varepsilon_{1}, \gamma_{1}\right)$ and $\left(\varepsilon_{2}, \gamma_{2}\right)$. Thus $\triangle_{\mathscr{E}} B$ satisfies (F2).

Since the forgetful functors from $\triangle_{\mathscr{C}} B$ to $B \downarrow$ we $\mathscr{E}$ and $\mathscr{E} \downarrow B$ are surjective both on objects and arrows, they preserve colimits. That is, we may rewrite localisations and colocalisations as colimits over $\triangle_{\mathscr{E}} B$.

THEOREM 4.2. Let $\mathscr{T}$ be a triangulated category and $\mathscr{E}$ a thick subcategory. Let $F: \mathscr{T} \rightarrow \mathfrak{U} \mathfrak{b}$ be a homological functor to the category of Abelian groups. Then there is a natural exact sequence

$$
\begin{aligned}
\cdots \rightarrow \mathrm{R}^{\perp} F_{1}(B) \rightarrow F_{1}(B) \rightarrow & \mathrm{R} F_{1}(B) \\
& \rightarrow \mathrm{R}^{\perp} F_{0}(B) \rightarrow F_{0}(B) \rightarrow \mathrm{R} F_{0}(B) \rightarrow \cdots
\end{aligned}
$$

This is the exact sequence (1) promised in the introduction.

Proof. For each object $E \rightarrow B \rightarrow C \rightarrow E$ [1] of $\triangle_{\mathscr{E}} B$, we get a long exact sequence

$$
\begin{aligned}
\cdots \rightarrow F_{1}(E) \rightarrow F_{1}(B) \rightarrow & F_{1}(C) \\
& \rightarrow F_{0}(E) \rightarrow F_{0}(B) \rightarrow F_{0}(C) \rightarrow \cdots
\end{aligned}
$$

This is a functor from $\triangle_{\mathscr{C}} B$ to the category of exact chain complexes in $\mathfrak{X} \mathfrak{b}$. Since $\triangle_{\mathscr{C}} B$ is filtered, the colimit of this diagram of chain complexes is again an exact chain complex. 
Since the forgetful functors from $\triangle_{\mathscr{E}} B$ to $B \downarrow$ we $_{\mathscr{E}}$ and $\mathscr{E} \downarrow B$ are surjective on objects and arrows, we have

$$
\mathrm{R} F(B) \cong \underset{\Delta_{\mathscr{E} B}}{\lim _{\longrightarrow}} F(C), \quad \mathrm{R}^{\perp} F(B) \cong \underset{\Delta_{\mathscr{E} B}}{\lim _{\longrightarrow}} F(E) .
$$

Since $\triangle_{\mathscr{E}} B$ is filtered, the colimit of the constant diagram $F(B)$ on $\triangle_{\mathscr{E}} B$ is $F(B)$. Hence the colimit of the exact sequences $(7)$ is the desired exact sequence (1).

Theorem 4.2 for the functor $\mathscr{T}(A, \sqcup)$ implies the exact sequence (2).

When we apply Theorem 4.2 to a homological functor $\mathscr{T}^{\text {op }} \rightarrow \mathfrak{U} \mathfrak{b}$, we get the exact sequence (1) for a cohomological functor $\mathscr{T} \rightarrow \mathfrak{H} \mathfrak{b}$.

COROLlARY 4.3. A homological or cohomological functor $F$ is local if and only if $\mathrm{R}^{\perp} F \cong 0$, and $F$ is colocal if and only if $\mathrm{R} F \cong 0$.

Corollary 4.4. A natural transformation $\Phi: F \Rightarrow F^{\prime}$ between two homological or cohomological functors is invertible if and only if both its localisation $\mathrm{R} \Phi: \mathrm{R} F \Rightarrow \mathrm{R} F^{\prime}$ and colocalisation $\mathrm{R}^{\perp} \Phi: \mathrm{R}^{\perp} F \Rightarrow \mathrm{R}^{\perp} F^{\prime}$ are invertible.

Proof. It is clear that invertibility of $\Phi$ implies invertibility of $R \Phi$ and $\mathrm{R}^{\perp} \Phi$. The converse follows from the Five Lemma and Theorem 4.2.

\section{REFERENCES}

1. Artin, Michael, and Zhang, James J., Noncommutative projective schemes, Adv. Math. 109 (1994), 228-287.

2. Bondal, A. I., and Kapranov, Mikhail M., Framed triangulated categories, Mat. Sb. 181 (1990), no. 5, 669-683 (Russian), translation in: Math. USSR-Sb. 70 (1991), 93-107.

3. Buchweitz, Ragnar-Olaf, Maximal Cohen-Macaulay modules and Tate cohomology over Gorenstein rings, unpublished preprint 1986, available at http: / / hdl. handle. net/1807/16682.

4. Gabriel, Peter, and Zisman, Michel, Calculus of Fractions and Homotopy Theory, Ergebnisse Math. Grenzgeb. 35, Springer, New York 1967.

5. Inassaridze, Hvedri, Kandelaki, Tamaz, and Meyer, Ralf, Localisation and colocalisation of KK-theory at sets of primes, Abh. Math. Semin. Univ. Hambg. 81 (2011), 19-34.

6. Kashiwara, Masaki, and Schapira, Pierre, Categories and Sheaves, Grundlehren Math. Wiss. 332, Springer, Berlin 2006.

7. Meyer, Ralf, and Nest, Ryszard, The Baum-Connes conjecture via localisation of categories, Topology 45 (2006), 209-259. 
8. Neeman, Amnon, Triangulated Categories, Annals of Math. Studies 148, Princeton Univ. Press, Princeton, NJ 2001.

\author{
A. RAZMADZE MATHEMATICAL INSTITUTE \\ TBILISI STATE UNIVERSITY \\ UNIVERSITY STREET 2 \\ TBILISI 380043 \\ GEORGIA \\ AND \\ TBILISI CENTRE FOR MATHEMATICAL SCIENCES \\ CHAVCHAVADZE AVE. 75, 3/35 \\ TBILISI 0168 \\ GEORGIA \\ E-mail: inassari@gmail.com \\ tam.kandel@gmail.com
}

\author{
MATHEMATISCHES INSTITUT \\ AND \\ COURANT CENTRE "HIGHER ORDER STRUCTURES" \\ GEORG-AUGUST UNIVERSITÄT GÖTTINGEN \\ BUNSENSTRASSE 3-5 \\ 37073 GÖTTINGEN \\ GERMANY \\ E-mail: rameyer@uni-math.gwdg.de
}

